\title{
Théorèmes ergodiques pour une translation sur un nilvariété
}

\author{
EMMANUEL LESIGNE
}

Faculté des Sciences et Techniques, 6 Av. Le Gorgeu, 29287, Brest Cedex, France

(Received 12 May 1987 and revised 24 September 1987)

Abstract. Let $T$ be a translation defined on a nil-manifold $N /{ }_{\Gamma}$, where $N$ is a nilpotent group of order two.

We prove two theorems:

- the first one asserts that the sequence

$$
\left(\frac{1}{n} \sum_{k=0}^{n-1} f\left(T^{k} x\right)\right)
$$

is convergent for all continuous functions $f$ on $N / \Gamma$ and all points $x$ of $N / \Gamma$.

- the second gives an explicit formula for the limit of

$$
\frac{1}{n} \sum_{k=0}^{n-1} f_{1}\left(T^{k} x\right) \cdot f_{2}\left(T^{2 k} x\right) \cdot f_{3}\left(T^{3 k} x\right)
$$

when $T$ is ergodic.

In the proof of the first result, we use an ergodic theorem due to Wiener and Wintner.

Résumé. Pour une translation $T$ définie sur un quotient compact $X$ de groupe nilpotent d'ordre 2, on démontre deux théorèmes:

- le premier affirme que la suite

$$
\left(\frac{1}{n} \sum_{k=0}^{n-1} f\left(T^{k} x\right)\right)
$$

converge pour toute fonction continue $f$ sur $X$ et tout point $x$ de $X$.

- le second donne une formule presque sûre explicite de la limite de la suite

$$
\frac{1}{n} \sum_{k=0}^{n-1} f_{1}\left(T^{k} x\right) \cdot f_{2}\left(T^{2 k} x\right) \cdot f_{3}\left(T^{3 k} x\right)
$$

quand $T$ est ergodique et $f_{1}, f_{2}$ et $f_{3}$ sont mesurables et bornées sur $X$.

On utilise, pour démontrer le premier résultat un théorème ergodique du à Wiener et Wintner.

\section{Introduction}

Soit $N$ un groupe de Lie nilpotent connexe et simplement connexe, et $\Gamma$ un sous-groupe discret de $N$ tel que $N /{ }_{\Gamma}$ soit compact (on note $N / \Gamma$ l'espace des classes à droite modulo $\Gamma$ ). On note $m$ la mesure de probabilité sur $N / \Gamma$ qui est l'image, par la projection de $N$ sur $N / \mathrm{r}$, de la mesure de Haar sur $N$, convenablement 
normalisée. Cette probabilité est invariante par l'action de $N$ sur $N / \mathrm{r}$. L'étude de ces 'nil-variétés' a été faite par Malce'v ([9], cf, également [13]). Soit a un point fixé de $N$. On note $T$ la transformation de $N / \Gamma$ induite par la translation à gauche par a sur $N$; ainsi, si $x \in N$, on a $T(x \cdot \Gamma)=a x \cdot \Gamma$. Cette 'nil-transformation' laisse la probabilité $m$ invariante. On a ainsi défini un système dynamique probabilisé $(N / \Gamma, m, T)$. Ce type de système a été étudié initialement par Auslander, Green et Hahn dans [1].

C'est l'étude de la convergence d'expressions de la forme

$$
\frac{1}{n} \cdot \sum_{k=0}^{n-1} f_{1}\left(\tau^{k} \omega\right) \cdot f_{2}\left(\tau^{2 k} \omega\right) \cdot f_{3}\left(\tau^{3 k} \omega\right)
$$

définies sur un système dynamique probabilisé $(\Omega, \mu, \tau)$ qui nous a conduit à l'énoncé de résultats sur les nil-transformations. Un théorème de convergence en moyenne pour les expressions (1) avait été énoncé dans [3], mais la démonstration comportait une erreur. Ceci a été remarqué par $\mathrm{H}$. Furstenberg et $\mathrm{B}$. Weiss, et ils ont noté le rôle particulier joué par les nil-transformations dans le comportement de (1). Nous disposons à présent d'une nouvelle preuve du théorème de convergence en moyenne de (1) et elle fera l'objet d'une prochaine publication; le théorème 1 du présent article intervient de façon essentielle dans la démonstration. La question de la convergence presque sûre de (1) est un problème non résolu en général; le théorème 1 permet d'affirmer que cette convergence a lieu quand $\tau$ est une nil-transformation et le Théorème 2 donne une expression pour la limite. Enfin une autre motivation pour l'étude du théorème 1 est la suivante: on sait, grâce à un exemple de Furstenberg dans [5], qu'il existe des systèmes dynamiques distaux et minimaux mais non uniquement ergodiques. Qu'en est-il des systèmes dynamiques distaux construits à partir d'une structure algébrique?

Revenons au système $(N / \Gamma, m, T)$ défini précédemment. Si $x$ et $y$ sont deux éléments de $N$, on pose $[x, y]=x y x^{-1} y^{-1}$. On note $N^{\prime}$ le sous-groupe dérivé de $N$, c'est à dire le sous-groupe engendré par $\{[x, y] / x, y \in N\}$. On sait que $N^{\prime}$ est fermé; le quotient $N / N^{\prime}$ est un groupe abélien et le quotient $N / \Gamma_{\text {. } N^{\prime}}$ est un groupe compact abélien. Rappelons les résultats de base sur l'ergodicité de la nil-transformation $T$.

La transformation $T$ induit une translation $R$ du groupe compact abélien $N / \Gamma \cdot N^{\prime}$. Le système $\left(N / \Gamma \cdot N^{\prime}, R\right)$ est un facteur du système $(N / \Gamma, T)$. Le théorème de Green affirme qu'il suffit que la translation $R$ soit ergodique pour que le système $(N / \Gamma, m, T)$ soit ergodique. Le théorème de Parry affirme qu'il suffit que le système $(N / \Gamma, m, T)$ soit ergodique pour que le système $(N / \Gamma, T)$ soit uniquement ergodique.

On trouvera ces résultats démontrés dans [1]; des démonstrations simples ont été données par Parry dans [11] et [12].

Grâce aux caractérisations connues sur l'ergodicité des translations de groupe compact abélien on peut résumer les théorèmes précédents par: il suffit que $\left\{a^{n} \gamma \mid n \in \mathbb{N}, \gamma \in \Gamma\right\}$ soit partout dense dans $N$ pour que la nil-transformation $T$ soit uniquement ergodique. Si c'est le cas, on sait que, pour toute fonction continue $f$ sur $N /{ }_{\Gamma}$, la suite

$$
\frac{1}{n} \cdot \sum_{k=0}^{n-1} f \circ T^{k}
$$


converge uniformément sur $N / \Gamma$. (La référence classique sur l'unique ergodicité est l'article d'Oxtoby [10].)

Nous nous intéressons particulièrement au problème suivant: si $f_{1}, f_{2}$ et $f_{3}$ sont des fonctions continues sur $N / \Gamma$, la suite

$$
\frac{1}{n} \sum_{k=0}^{n-1} f_{1} \circ T^{k} \cdot f_{2} \circ T^{2 k} \cdot f_{3} \circ T^{3 k}
$$

converge-t-elle et, si oui, que peut-on dire de sa limite? Si la transformation $T \times T^{2} \times T^{3}$ définie sur $N / \Gamma_{\Gamma} \times N / \Gamma \times N / \Gamma$ était uniquement ergodique la convergence serait assurée et la limite connue, mais cette transformation produit n'est jamais ergodique car le système $(N / \Gamma, m, T)$ a un facteur à spectre discret non trivial. C'est la raison pour laquelle nous avons été amenés à étudier la convergence simple de moyennes du type

$$
\frac{1}{n} \sum_{k=0}^{n-1} f \circ T^{k}
$$

sans hypothèse d'ergodicité.

L'auteur tient à remercier J. P. Conze pour l'aide qu'il lui a apportée dans ce travail, au cours de nombreuses et fructueuses conversations.

Nous allons, dans toute la suite, faire l'hypothèse que le groupe dérivé $N^{\prime}$ de $N$ est contenu dans le centre de $N$; autrement dit, on suppose que la série centrale descendante de $N$ se réduit à $N \supset N^{\prime}=[N, N] \supset N^{\prime \prime}=\left[N^{\prime}, N\right]=\{e\}$; un tel groupe est dit nilpotent d'ordre 2.

Donnons un exemple satisfaisant les diverses hypothèses: soient $p$ et $q$ deux entiers strictement positifs, et $B$ une application bilinéaire de $\mathbb{R}^{p} \times \mathbb{R}^{p}$ dans $\mathbb{R}^{q}$. On pose $N=\mathbb{R}^{p}+\mathbb{R}^{q}$ muni de la loi suivante: si $x, y \in \mathbb{R}^{p}$ et $x^{\prime}, y^{\prime} \in \mathbb{R}^{q}$, alors

$$
\left(x, x^{\prime}\right) \cdot\left(y, y^{\prime}\right)=\left(x+y, x^{\prime}+y^{\prime}+B(x, y)\right) .
$$

Le groupe dérivé $N^{\prime}$ est un sous-groupe de $\mathbb{R}^{q}$. Si on suppose que $B$ est à coefficients entiers alors $\Gamma=\mathbb{Z}^{p} \times \mathbb{Z}^{q}$ est un sous-groupe discret de $N$ et le quotient $N / \Gamma$ est compact. On peut de plus choisir $B$ de façon que $N^{\prime}$ soit égal à $\mathbb{R}^{q}$. On a alors: le groupe abélien $N / N^{\prime}$ est $\mathbb{R}^{p}$, le groupe compact abélien $N / \Gamma_{N^{\prime}}$ est le tore $(\mathbb{R} / z)^{p}$; le groupe compact abélien $N^{\prime} / \Gamma \cap N^{\prime}$ est le tore $(\mathbb{R} / \mathbf{Z})^{q}$.

\section{Enonce des resultats}

Tous les résultats énoncés dans ce paragraphe seront démontrés dans la suite.

THÉORÈME 1. Pour tous a et $x$ dans $N$ et toute fonction continue $f$ sur $N / \Gamma$, la suite

$$
\frac{1}{n} \sum_{k=0}^{n-1} f\left(a^{k} x \cdot \Gamma\right)
$$

est convergente

Remarques. Pour tout $x$ dans $N$, notons $O_{x}=\overline{\left\{a^{n} x \cdot \Gamma \mid n \in \mathbb{Z}\right\}}$.

(a) Un énoncé équivalent au Théorème 1 est le suivant: pour tout $x$ dans $N$, le système dynamique $\left(O_{x}, T\right)$ est uniquement ergodique. (Justifions rapidement cette remarque. Le système $(N / \Gamma, T)$ est distal et donc, d'après Furstenberg ([6], Théorème 3.2)), la fermeture de l'orbite d'un point quelconque définit un système 
minimal. D'autre part, on trouve dans Oxtoby ([10], Proposition (5.4)) une preuve du fait suivant: si $(X, T)$ est un système minimal tel que les moyennes ergodiques associées à toute fonction continue convergent en tout point, alors ce système est uniquement ergodique).

(b) On sait que toute nil-transformation minimale est uniquement ergodique. $\mathrm{Si}$ la restriction de la nil-transformation $T$ aux ensembles minimaux $O_{x}$ était elle-même une nil-transformation, le théorème de convergence serait donc acquis. Considérons l'orbite fermée $O_{e}$ de l'élément neutre de $N ; O_{e}=\left\{a^{n} \Gamma \mid n \in \mathbb{Z}\right\}$. Contrairement à ce que l'on pourrait espérer, il n'existe pas en général de sous-groupe fermé $M$ de $N$, contenant $\Gamma$ et tel que $O_{e}=M / \Gamma$. De fait, si on note $M$ le plus petit sous-groupe fermé de $N$ contenant a et $\Gamma$, l'espace homogène $M / \Gamma$ peut être plus gros que $O_{e}$. Donnons un exemple: soit $N$ le groupe d'Heisenberg,

$$
N=\left\{\left(\begin{array}{ccc}
1 & x_{1} & x_{3} \\
0 & 1 & x_{2} \\
0 & 0 & 1
\end{array}\right) \mid x_{1}, x_{2}, x_{3} \in \mathbb{R}\right\}
$$

soient

$$
\Gamma=\left\{\left(\begin{array}{ccc}
1 & n_{1} & n_{3} \\
0 & 1 & n_{2} \\
0 & 0 & 1
\end{array}\right) n_{1}, n_{2}, n_{3} \in \mathbb{Z}\right\} \quad \text { et } \quad a=\left(\begin{array}{ccc}
1 & 0 & 0 \\
0 & 1 & a_{2} \\
0 & 0 & 1
\end{array}\right)
$$

où $a_{2}$ est un nombre irrationnel; on a alors

$$
M=\left\{\left(\begin{array}{ccc}
1 & n_{1} & x_{3} \\
0 & 1 & x_{2} \\
0 & 0 & 1
\end{array}\right) \mid n_{1} \in \mathbb{Z} \text { et } x_{2}, x_{3} \in \mathbb{R}\right\}
$$

et donc $M / \Gamma$ est de dimension 2 ;

$$
O_{e}=\left\{\left(\begin{array}{ccc}
1 & n_{1} & n_{3} \\
0 & 1 & x_{2} \\
0 & 0 & 1
\end{array}\right) \mid n_{1}, n_{3} \in \mathbb{Z} \text { et } x_{2} \in \mathbb{R}\right\} \Gamma
$$

et donc $O_{e}$ est de dimension 1.

(Il est à noter que, pour presque tout $x$, l'orbite $O_{x}$ est de dimension 2. Ainsi il existe des valeurs exceptionnelles du point $x$ pour lesquelles la dimension de l'orbite chute).

On déduira du Théorème 1 les deux corollaires suivants:

Corollaire 1. Pour tous a et $x$ dans $N$, pour toutes fonctions continues $f_{1}, f_{2}$ et $f_{3}$ sur $N / \Gamma$, la suite

$$
\frac{1}{n} \sum_{k=0}^{n-1} f_{1}\left(a^{k} x \cdot \Gamma\right) \cdot f_{2}\left(a^{2 k} x \cdot \Gamma\right) \cdot f_{3}\left(a^{3 k} x \cdot \Gamma\right) \quad \text { est convergente. }
$$

Corollaire 2. Pour tout a dans $N$, pour toutes fonctions mesurables bornées $f_{1}, f_{2}$ et $f_{3}$ sur $N / \Gamma$, la suite

$$
\left.\frac{1}{n} \sum_{k=0}^{n-1} f_{1}\left(a^{k} x \cdot \Gamma\right) \cdot f_{2}\left(a^{2 k} x \cdot \Gamma\right) \cdot f_{3}\left(a^{3 k} x \cdot \Gamma\right)\right)
$$

converge en presque tout point $x$ de $N$. 
Nous noterons $\Gamma^{\prime}=\Gamma \cap N^{\prime}$; c'est un sous-groupe discret de $N^{\prime}$. Grâce aux travaux de Malce'v, on sait que l'espace homogène $N^{\prime} / \Gamma^{\prime}$ est compact. On note $m^{\prime}$ la mesure de probabilité sur $N^{\prime} / \Gamma^{\prime}$ qui est invariante sous l'action de $N^{\prime}$. En fait, puisque l'on a supposé que le groupe $N$ était nilpotent d'ordre 2, l'espace $N^{\prime} / \Gamma^{\prime}$ est un groupe compact abélien et $m^{\prime}$ est sa mesure de Haar normalisée. Enonçons le second théorème.

THÉORÈME 2. On suppose que la nil-transformation T est ergodique. On a alors: pour toutes fonctions $f_{1}, f_{2}$ et $f_{3}$ mesurables et bornées sur $N /_{\Gamma}$ et pour presque tout $x$ dans $N$,

$$
\begin{aligned}
\lim _{n \rightarrow+\infty} & \frac{1}{n} \sum_{k=0}^{n-1} f_{1}\left(a^{k} x \cdot \Gamma\right) \cdot f_{2}\left(a^{2 k} x \cdot \Gamma\right) \cdot f_{3}\left(a^{3 k} x \cdot \Gamma\right) \\
\quad= & \int_{N / \Gamma} \int_{N^{\prime} / \Gamma} f_{1}(x y \cdot \Gamma) \cdot f_{2}\left(x y^{2} z \cdot \Gamma\right) \cdot f_{3}\left(x y^{3} z^{3} \cdot \Gamma\right) d m(y \cdot \Gamma) d m^{\prime}\left(z \cdot \Gamma^{\prime}\right) .
\end{aligned}
$$

Remarquons que la forme de la limite peut être partiellement justifiée par le fait que l'ensemble $\left\{\left(y, y^{2} z, y^{3} z^{3}\right) \mid y \in N, z \in N^{\prime}\right\}$ est le plus petit sous-groupe $H$ de $N^{3}$ vérifiant: si $\left(x_{1}, x_{2}, x_{3}\right) \in H$ et $y \in N$, alors $\left(y x_{1}, y^{2} x_{2}, y^{3} x_{3}\right) \in H$.

\section{Un theoreme ergodique de Wiener et Wintner}

Nous allons, pour démontrer le Théorème 1, utiliser le résultat suivant:

Théorème DE WiEner ET Wintner. Soit $(\Omega, \mathscr{C}, \mu)$ un espace probabilisé de Lebesgue, et $\tau$ une transformation bijective, bimesurable de cet espace conservant la probabilité $\mu$. Si $f \in \mathscr{L}^{1}(\mu)$, on a, pour $\mu$-presque tout $\omega$ : pour tout z nombre complexe de module 1 , la suite

$$
\frac{1}{n} \sum_{k=0}^{n-1} z^{k} \cdot f\left(\tau^{k} \omega\right) \quad \text { converge. }
$$

Ce théorème généralise le théorème ergodique de Birkhoff. En effet celui-ci permet d'affirmer (en l'appliquant à un système produit) que, pour tout $f \in \mathscr{L}^{\prime}(\mu)$ et tout $z$ complexe de module 1 , la suite

$$
\frac{1}{n} \sum_{k=0}^{n-1} z^{k} \cdot f\left(\tau^{k} \omega\right) \text { converge pour } \mu \text {-presque tout } \omega \text {. }
$$

La démonstration originale de ce théorème se trouve dans [14]. On peut donner une preuve rapide de ce résultat en utilisant la notion de systèmes dynamiques disjoints développée par Furstenberg dans [7]. Nous allons donner les grandes lignes d'une telle preuve. Notons auparavant que des démonstrations de ce théorème se trouvent dans Bellow-Losert [2] et, comme nous l'a justement fait remarquer le referee de cet article, dans la thèse de Furstenberg [4] (avec des idées qui sont très proches de celles ci-après résumées.)

Définition. Deux systèmes dynamiques probabilisés $(\Omega, \mathscr{A}, \mu, \tau)$ et $\left(\Omega^{\prime}, \mathscr{A}^{\prime}, \mu^{\prime}, \tau^{\prime}\right)$ sont disjoints si la mesure produit $\mu \times \mu^{\prime}$ est l'unique mesure sur $\left(\Omega \times \Omega^{\prime}, \mathscr{A} \times \mathscr{A}^{\prime}\right)$ 
invariante par $\tau \times \tau^{\prime}$ dont les projections sur $(\Omega, \mathscr{A})$ et $\left(\Omega^{\prime}, \mathscr{A}^{\prime}\right)$ coïncident avec $\mu$ et $\mu^{\prime}$ respectivement.

On considère à présent des systèmes dynamiques probabilisés réguliers, c'est à dire des systèmes $(\Omega, \mathscr{A}, \mu, \tau)$, où $\Omega$ est un espace compact métrique, $\mathscr{A}$ est sa tribu borélienne, $\mu$ est une probabilité régulière sur $(\Omega, \mathscr{A})$ et $\tau$ est un homéomorphisme de $\Omega$, conservant $\mu$. Modulo un théorème de représentation, c'est la situation générale (cf, [7]; appendice de la partie 1).

On note $\mathscr{C}(\Omega)$ l'espace des fonctions complexes continues sur $\Omega$. On appelle $\Omega_{1}$ l'ensemble des points génériques du système, c'est à dire

$$
\left\{\omega \in \Omega \mid \text { pour tout } f \in \mathscr{C}(\Omega), \quad \lim _{n \rightarrow+\infty} \frac{1}{n} \sum_{k=0}^{n-1} f\left(\tau^{k} \omega\right)=\int f d \mu\right\} .
$$

Si le système est ergodique, on a $\mu\left(\Omega_{1}\right)=1$ (c'est une conséquence de la séparabilité de $\mathscr{C}(\Omega)$ ).

ThÉOREME. ([7], theorem 1.6.) Soient $(\Omega, \mathscr{A}, \mu, \tau)$ et $\left(\Omega^{\prime}, \mathscr{A}^{\prime}, \mu^{\prime}, \tau^{\prime}\right)$ deux systèmes réguliers ergodiques disjoints. Tout point $\left(\omega, \omega^{\prime}\right)$ de $\Omega_{1} \times \Omega_{1}^{\prime}$ est un point générique pour le système produit $\left(\Omega \times \Omega^{\prime}, \mathscr{A} \times \mathscr{A}^{\prime}, \mu \times \mu^{\prime}, \tau \times \tau^{\prime}\right)$.

Proposition. Si $z$ est un nombre complexe de module 1 , on note $G_{z}$ le sous-groupe multiplicatif fermé engendré par $z$, on note $R_{z}$ la rotation $u \mapsto u z$ sur $G_{z}$ et on note $m_{z}$ la probabilité de Haar sur $G_{z}$. Soit $(\Omega, \mathscr{A}, \mu, \tau)$ un système régulier ergodique et $U_{\tau}$ l'opérateur de $\mathscr{L}^{2}(\mu)$ induit par $\tau$. On a: si, pour tout entier p non nul, $z^{p} n^{\prime}$ 'est pas une valeur propre de $U_{\tau}$, alors les systèmes $(\Omega, \mathscr{A}, \mu, \tau)$ et $\left(G_{z}, m_{z}, R_{z}\right)$ sont disjoints.

La preuve de cette proposition est basée sur le fait que, sous les hypothèses énoncées, le système produit $\left(\Omega \times G_{z}, \mu \times m_{z}, \tau \times R_{z}\right)$ est ergodique. Soit $(\Omega, \mathscr{A}, \mu, \tau)$ un système dynamique régulier ergodique. En associant la proposition et le théorème précédents, on obtient: si $z$ est un nombre complexe de module 1 dont aucune puissance $z^{p}(p \neq 0)$ n'est valeur propre de $U_{\tau}$, alors, pour tout $f$ dans $\mathscr{C}(\Omega)$ et tout $\omega$ dans $\Omega_{1}$,

$$
\lim _{n \rightarrow+\infty} \frac{1}{n} \sum_{k=0}^{n-1} z^{k} \cdot f\left(\tau^{k} \omega\right)=0
$$

Cette condition est vérifiée par tous les nombres complexes de module 1, en dehors d'un ensemble dénombrable $\Lambda$. Pour les éléments $z$ de $\Lambda$ on peut appliquer la remarque (2). On en déduit que, pour tout $f$ dans $\mathscr{C}(\Omega)$, pour $\mu$-presque tout $\omega$, on a: pour tout $z$ complexe de module 1

$$
\text { la suite }\left(\frac{1}{n} \sum_{k=0}^{n-1} z^{k} \cdot f\left(\tau^{k} \omega\right)\right) \text { converge. }
$$

Ce résultat, acquis pour les fonctions continues, s'étend par densité à toutes les fonctions $f$ intégrables en utilisant l'inégalité maximale 'uniforme en $z$ ' suivante:

$$
\mu\left(\left\{\omega: \sup _{\substack{n>0 \\|z|=1}} \frac{1}{n}\left|\sum_{k=0}^{n-1} z^{k} \cdot f\left(\tau^{k} \omega\right)\right|>\lambda\right\}\right) \leq \frac{\|f\|_{1}}{\lambda} .
$$


Pour démontrer le théorème dans toute sa généralité, il faut encore supprimer l'hypothèse d'ergodicité. Ceci se fait par le procédé usuel de désintégration d'une probabilité en barycentre de probabilités ergodiques.

Le théorème étant ainsi rapidement justifié, énonçons le corollaire qui nous servira dans la suite.

Corollaire 3. Soit $\Omega$ un espace métrique compact et $\tau$ un homéomorphisme de $\Omega$. Il existe un point $\omega_{0}$ dans $\Omega$ tel que, pour toute fonction continue $f$ sur $\Omega$ et pour tout $z$ complexe de module 1 , la suite

$$
\frac{1}{n} \sum_{k=0}^{n-1} z^{k} \cdot f\left(\tau^{k} \omega_{0}\right) \quad \text { converge. }
$$

Preuve $d u$ Corollaire 3. Il existe une probabilité $\mu$ sur $\Omega$, invariante par $\tau$. Le théorème de Wiener et Wintner nous affirme que: pour tout $f$ dans $\mathscr{C}(\Omega)$ et pour $\mu$-presque tout $\omega$, pour tout $z$ tel que $|z|=1$, la suite

$$
\left(\frac{1}{n} \sum_{k=0}^{n-1} z^{k} f\left(\tau^{k} \omega\right)\right) \text { converge. }
$$

Du fait que $\mathscr{C}(\Omega)$ contient une partie dénombrable dense pour la convergence uniforme, on en déduit, par des procédés usuels, que: pour $\mu$-presque tout $\omega$, pour tout $f$ dans $\mathscr{C}(\Omega)$ et pour tout $z$ tel que $|z|=1$, la suite

$$
\frac{1}{n} \sum_{k=0}^{n-1} z^{k} \cdot f\left(\tau^{k} \omega\right) \quad \text { converge. }
$$

Cette propriété vérifiée par $\mu$-presque tout $\omega$ est vérifiée par au moins un point $\omega_{0}$ de $\Omega$. Le corollaire est démontré.

\section{Démonstration du Théorème 1 et de ses corollaires}

On considère la transformation $T$ définie sur la nil-variété $N / \Gamma$. On suppose que le groupe $N$ est nilpotent d'ordre 2 .

On note $\Gamma^{\prime}=N^{\prime} \cap \Gamma$ et, si $X$ est un espace compact, on note $\mathscr{C}(X)$ l'espace des fonctions continues sur $X$, muni de la topologie de la convergence uniforme. Nous allons introduire un sous-espace dense de $\mathscr{C}(\mathrm{N} / \mathrm{r})$. Nous noterons $A$ l'ensemble des éléments $f$ de $\mathscr{C}(N / \Gamma)$ pour lesquels il existe un caractère $\sigma$ du groupe compact abélien $N^{\prime} / \Gamma^{\prime}$ tel que:

$$
\text { si } x \in N \text { et } x^{\prime} \in N^{\prime} \text {, alors } f\left(x^{\prime} x \cdot \Gamma\right)=\sigma\left(x^{\prime} \cdot \Gamma^{\prime}\right) \cdot f(x \cdot \Gamma) \text {. }
$$

Proposition. L'ensemble des combinaisons linéaires d'éléments de A est une sousalgèbre dense de $\mathscr{C}(\mathrm{N} / \mathrm{r})$.

Preuve de la proposition. Cette proposition est un cas particulier du résultat général suivant: soit $X$ un espace métrisable et $G$ un groupe compact abélien agissant continuement sur $X$. Notons $A$ l'ensemble des fonctions continues sur $X$ vérifiant: il existe $\sigma$ caractère de $G$ tel que, pour tout $g \in G$ et tout $x \in X$,

$$
f(g x)=\sigma(g) \cdot f(x) \text {. }
$$

On a alors: l'ensemble des combinaisons linéaires d'éléments de $A$ est une sousalgèbre dense de $\mathscr{C}(X)$. 
Démontrons ce résultat; l'ensemble des combinaisons linéaires d'éléments de $A$ est bien sûr une algèbre stable par conjugaison. Pour montrer que cette algèbre est dense dans $\mathscr{C}(X)$, il suffit, d'après le théorème de Stone-Weierstrass, de démontrer que $A$ sépare les points de $X$. Soient $x$ et $y$ deux points distincts de $X$. Nous noterons $\Pi$ la projection canonique de $X$ sur $X / G$.

Examinons tout d'abord le cas où $\Pi(x) \neq \Pi(y)$. Il existe alors une fonction continue $\tilde{f}$ sur $X / G$ telle que $\tilde{f}(\Pi(x)) \neq \tilde{f}(\Pi(y))$. On pose, pour tout $z$ dans $X, f(z)=\tilde{f}(\mathrm{II}(x))$. La fonction $f$ ainsi définie est dans $A$ et vérifie $f(x) \neq f(y)$. Examinons à présent le cas où $\Pi(x)=\Pi(y)$. Soit $g_{0} \in G$ tel que $g_{0} x=y$, et soit $\sigma$ un caractère de $G$ tel que $\sigma\left(g_{0}\right) \neq 1$. Du fait que $\{g x \mid g \in G\}$ est une partie compacte de l'espace métrisable $X$, on déduit que toute fonction continue sur $\{g x \mid g \in G\}$ se prolonge en une fonction continue sur $X$. En particulier, il existe une fonction continue $\tilde{f}$ sur $X$ telle que, pour tout $g$ dans $G, \tilde{f}(g x)=\sigma(g)$. On définit une fonction $f$ sur $X$ en posant

$$
\text { si } z \in X, \quad f(z)=\int_{G} \tilde{f}(g z) \bar{\sigma}(g) d g
$$

(où dg désigne la measure de Haar normalisée sur $G$ ). On a alors: $f \in A, f(x)=1$, $f(y)=\sigma\left(g_{0}\right) \neq 1$. Nous avons démontré que $A$ sépare les points de $X$, ce qui achève la preuve de la proposition.

Nous pouvons donner à présent la fin de la démonstration du Théorème 1. On fixe un point $a$ de $N$. Pour montrer que la suite

$$
\left(\frac{1}{n} \sum_{k=0}^{n-1} f\left(a^{k} x \cdot \Gamma\right)\right)
$$

converge pour tout $f$ dans $\mathscr{C}(N / \Gamma)$ et tout $x$ dans $N$, il suffit, grâce à la proposition précédente, de le démontrer pour tout $f$ dans $A$ et tout $x$ dans $N$. On déduit du Corollaire 3 qu'il existe $x_{0}$ dans $N$ tel que, pour tout $g \in \mathscr{C}(N / \Gamma)$ et tout $z$ complexe de module 1,

$$
\text { la suite }\left(\frac{1}{n} \sum_{k=0}^{n-1} z^{k} \cdot g\left(a^{k} x_{0} \cdot \Gamma\right)\right) \text { converge. }
$$

On fixe un tel point $x_{0}$. Soit $f \in A$ et $\sigma$ le caractère de $N^{\prime} / \Gamma^{\prime}$ tel que $f\left(x^{\prime} y \cdot \Gamma\right)=$ $\sigma\left(x^{\prime} \cdot \Gamma^{\prime}\right) \cdot f(y \cdot \Gamma)$ pour $y \in N$ et $x^{\prime} \in N^{\prime}$. Soit $x \in N$. On a:

$$
\frac{1}{n} \sum_{k=0}^{n-1} f\left(a^{k} x \cdot \Gamma\right)=\frac{1}{n} \sum_{k=0}^{n-1} f\left(a^{k} x x_{0}^{-1} x_{0} \cdot \Gamma\right)=\frac{1}{n} \sum_{k=0}^{n-1} f\left(\left[a^{k}, x x_{0}^{-1}\right] x x_{0}^{-1} a^{k} x_{0} \cdot \Gamma\right) .
$$

Le groupe $N$ étant nilpotent d'ordre 2 , on a $\left[a^{k}, x x_{0}^{-1}\right]=\left[a, x x_{0}^{-1}\right]^{k}$. On en déduit que:

$$
\begin{aligned}
\frac{1}{n} \sum_{k=0}^{n-1} f\left(a^{k} x \cdot \Gamma\right) & =\frac{1}{n} \sum_{k=0}^{n-1} \sigma\left(\left[a^{k}, x x_{0}^{-1}\right]\right) \cdot f\left(x x_{0}^{-1} a^{k} x_{0} \cdot \Gamma\right) \\
& =\frac{1}{n} \sum_{k=0}^{n-1}\left(\sigma\left(\left[a, x x_{0}^{-1}\right]\right)\right)^{k} \cdot f\left(x x_{0}^{-1} a^{k} x_{0} \cdot \Gamma\right) \\
& =\frac{1}{n} \sum_{k=0}^{n-1} z^{k} \cdot g\left(a^{k} x_{0} \cdot \Gamma\right)
\end{aligned}
$$


où $z=\sigma\left(\left[a, x x_{0}^{-1}\right]\right)$ et la fonction $g$ est définie par $g(y \cdot \Gamma)=f\left(x x_{0}^{-1} y \cdot \Gamma\right)$, pour tout $y \in N$. Ceci prouve que la suite

$$
\left(\frac{1}{n} \sum_{k=0}^{n-1} f\left(a^{k} x \cdot \Gamma\right)\right) \text { converge, }
$$

et le théorème est démontré.

Pour justifier le corollaire 1, il suffit de remarquer que le Théorème 1 s'applique à la nil-variété $N \times N \times N / \Gamma \times \Gamma \times \Gamma$, munie de la nil-transformation $T \times T^{2} \times T^{3}$, induite par le point $\left(a, a^{2}, a^{3}\right)$ de $N \times N \times N$.

Le Corollaire 2 se déduit du précédent grâce à une inégalité maximale qui a déjà été utilisée plusieurs fois dans l'étude de la convergence presque sûre des moyennes de la forme

$$
\frac{1}{n} \sum_{k=0}^{n-1} f_{1} \circ T^{k} \cdot f_{2} \circ T^{2 k} \cdots f_{p} \circ T^{p k}
$$

(cf, [8], ou [3] - Lemme 3, p. 173).

\section{Démonstration du Théorème 2}

On définit un groupe $(M, *)$ par: $M=N \times N^{\prime}$ et, si $x, y \in N$ et $x^{\prime}, y^{\prime} \in N^{\prime}$, $\left(x, x^{\prime}\right) *\left(y, y^{\prime}\right)=\left(x y, x^{\prime} y^{\prime}[x, y]\right)$. Nous allons prouver que

$M$ est un groupe de Lie nilpotent connexe et simplement connexe.

$\left(\Gamma \times \Gamma^{\prime}, *\right)$ est un sous-groupe discret de $M$ et le quotient

$$
M / \Gamma \times \Gamma^{\prime} \text { est compact. }
$$

On note e l'élément neutre du groupe $N$.

On munit l'espace $M / \Gamma \times \Gamma^{\prime}$ de l'unique probabilité invariante sous l'action de $M$, et on considère, pour tout $x$ dans $N$, la transformation $S_{x}$ de $M /_{\Gamma \times \Gamma^{\prime}}$ induite par la translation à gauche sur $M$ par l'élément $(a[a, x], e)$. Ainsi, si $\left(y, y^{\prime}\right) \in M$, on a:

$$
S_{x}\left(\left(y, y^{\prime}\right) *\left(\Gamma \times \Gamma^{\prime}\right)\right)=\left(a y[a, x],[a, y] y^{\prime}\right) *\left(\Gamma \times \Gamma^{\prime}\right) \text {. }
$$

Nous allons prouver, sous l'hypothèse de l'ergodicité de $T$, que:

(4) pour presque tout $x$ dans $N$, la transformation $S_{x}$ est ergodique.

Avant de donner des preuves des affirmations (3) et (4), donnons une démonstration du théorème. Elle est basée sur la remarque suivante: soit $x \in N$; notons $A_{x}$ la fermeture de l'orbite du point $(x \cdot \Gamma, x \cdot \Gamma, x \cdot \Gamma)$ sous la transformation $T \times T^{2} \times T^{3}$; les systèmes dynamiques topologiques $\left(A_{x}, T \times T^{2} \times T^{3}\right)$ et $\left(M / \Gamma \times \Gamma^{\prime}, S_{x}\right)$ sont isomorphes. Donnons le détail de cet argument.

Fixons le point $x$ de $N$. Considérons l'application $I$ de $M$ dans $(N / \Gamma)^{3}$ définie par $I\left(y, y^{\prime}\right)=\left(x y \cdot \Gamma, x y^{2} y^{\prime} \cdot \Gamma, x y^{3} y^{\prime 3} \cdot \Gamma\right)$. Cette application est continue. Soit $\left(\gamma, \gamma^{\prime}\right) \in \Gamma \times \Gamma^{\prime} ;$ on a, pour tout $\left(y, y^{\prime}\right)$ dans $M$,

$$
\begin{aligned}
I\left(\left(y, y^{\prime}\right) *\left(\gamma, \gamma^{\prime}\right)\right) & =I\left(y \gamma, y^{\prime} \gamma^{\prime}[y, \gamma]\right) \\
& =\left(x y \gamma \cdot \Gamma, x(y \gamma)^{2}[y, \gamma] y^{\prime} \gamma^{\prime} \cdot \Gamma, x(y \gamma)^{3}[y, \gamma]^{3} y^{\prime 3} \gamma^{\prime 3} \cdot \Gamma\right) \\
& =\left(x y \gamma \cdot \Gamma, x y^{2} y^{\prime} \gamma^{2} \gamma^{\prime} \cdot \Gamma, x y^{3} y^{\prime 3} \gamma^{3} \gamma^{\prime 3} \cdot \Gamma\right) \\
& =\left(x y \cdot \Gamma, x y^{2} y^{\prime} \cdot \Gamma, x y^{3} y^{\prime 3} \cdot \Gamma\right) \\
& =I\left(y, y^{\prime}\right) .
\end{aligned}
$$


(Rappelons que les éléments de $N^{\prime}$ commutent avec ceux de $N$.) Ainsi $I$ est en fait une application de $M / \Gamma \times \Gamma^{\prime}$ dans $(N / \Gamma)^{3}$. Soit $\left(y, y^{\prime}\right) \in M$; on $a$ :

$$
\begin{aligned}
& I\left(S_{x}\left(\left(y, y^{\prime}\right) *\left(\Gamma \times \Gamma^{\prime}\right)\right)\right) \\
& \quad=I\left(\left(a y[a, x],[a, y] y^{\prime}\right) *\left(\Gamma \times \Gamma^{\prime}\right)\right) \\
& \quad=\left(x a y[a, x] \cdot \Gamma, x(a y)^{2}[a, x]^{2}[a, y] y^{\prime} \cdot \Gamma, x(a y)^{3}[a, x]^{3}[a, y]^{3} y^{\prime 3} \cdot \Gamma\right) \\
& \quad=\left(a x y \cdot \Gamma, a^{2} x y^{2} y^{\prime} \cdot \Gamma, a^{3} x y^{3} y^{\prime 3} \cdot \Gamma\right)=\left(T \times T^{2} \times T^{3}\right)\left(I\left(\left(y, y^{\prime}\right) *\left(\Gamma \times \Gamma^{\prime}\right)\right)\right) .
\end{aligned}
$$

Autrement dit: $I \circ S_{x}=\left(T \times T^{2} \times T^{3}\right) \circ I$. Notons $m^{\prime \prime}$ la probabilité sur $M / \Gamma \times \Gamma^{\prime}$ invariante sous l'action de $M$. Notons $\mu_{x}$ l'image de $m^{\prime \prime}$ par $I$. La probabilité $m^{\prime \prime}$ est la probabilité produit $m \times m^{\prime}$, et, si $F$ est une fonction continue $\operatorname{sur}(N / \Gamma)^{3}$, on a

$$
\int F d \mu_{x}=\int_{N / \Gamma} \int_{N^{\prime} / \Gamma^{\prime}} F\left(x y \cdot \Gamma, x y^{2} y^{\prime} \cdot \Gamma, x y^{3} y^{\prime 3} \cdot \Gamma\right) d m(y \cdot \Gamma) d m^{\prime}\left(y^{\prime} \cdot \Gamma^{\prime}\right) .
$$

Notons $A_{x}=\left\{\left(x y \cdot \Gamma, x y^{2} y^{\prime} \cdot \Gamma, x y^{3} y^{\prime 3} \cdot \Gamma\right) \mid y \in N\right.$ et $\left.y^{\prime} \in N^{\prime}\right\}$. L'application $I$ est un isomorphisme entre les systèmes dynamiques mesurés et topologiques $\left(A_{x}, \mu_{x}, T \times\right.$ $\left.T^{2} \times T^{3}\right)$ et $\left(M /{ }_{\Gamma \times \Gamma^{\prime}}, m^{\prime \prime}, S_{x}\right)$. Si $x$ est tel que le système $\left(M / \Gamma \times \Gamma^{\prime}, m^{\prime \prime}, S_{x}\right)$ est ergodique, alors, d'après le théorème de Parry, ce système est uniquement ergodique, et donc, d'après l'isomorphisme précédent, le système $\left(A_{x}, \mu_{x}, T \times T^{2} \times T^{3}\right)$ est uniquement ergodique. Pour un tel $x$, on a: pour tout $\left(y, y^{\prime}\right)$ dans $N \times N^{\prime}$, pour tout $F \in \mathscr{C}\left((N / \Gamma)^{3}\right)$,

$$
\lim _{n \rightarrow+\infty} \frac{1}{n} \sum_{k=0}^{n-1} F\left(a^{k} x y \cdot \Gamma, a^{2 k} x y^{2} y^{\prime} \cdot \Gamma, a^{3 k} x y^{3} y^{3} \cdot \Gamma\right)=\int F d \mu_{x}
$$

En particulier, pour tout $F \in \mathscr{C}\left((N / \Gamma)^{3}\right)$,

$$
\lim _{n \rightarrow+\infty} \frac{1}{n} \sum_{k=0}^{n-1} F\left(a^{k} x \cdot \Gamma, a^{2 k} x \cdot \Gamma, a^{3 k} x \cdot \Gamma\right)=\int F d \mu_{x}
$$

D'après (4), ceci est vrai pour presque tout $x$ dans $N$. Ainsi le Théorème 2 est démontré quand $f_{1}, f_{2}$, et $f_{3}$ sont des fonctions continues. Le cas où $f_{1}, f_{2}$ et $f_{3}$ sont seulement supposées mesurables et bornées se traite sans difficulté par approximations en utilisant l'inégalité maximale déjà évoquée dans le paragraphe précédent.

La preuve du théorème sera achevée quand les affirmations (3) et (4) auront été démontrées.

Preuve de (3). En tant qu'espace topologique et variété différentiable, l'ensemble $M$ est le produit $N \times N^{\prime}$. Le fait que $M$ soit un groupe de Lie nilpotent connexe et simplement connexe et le fait que $\Gamma \times \Gamma^{\prime}$ soit un sous-groupe discret de $M$ sont donc évidents. Il nous faut montrer que le quotient $M /_{\Gamma \times \Gamma^{\prime}}$ est compact. Considérons une suite $\left(x_{n}, x_{n}^{\prime}\right)$ dans $M$. Puisque l'espace $N / r$ est compact, on peut trouver une suite croissante d'entiers $\left(n_{h}\right)_{h \geq 0}$ et une suite $\left(\gamma_{n_{h}}\right)$ dans $\Gamma$ telles que la suite $\left(x_{n_{h}} \cdot \gamma_{n_{h}}\right)$ converge dans $N$.

On a

$$
\left(x_{n_{h}}, x_{n_{h}}^{\prime}\right) *\left(\Gamma \times \Gamma^{\prime}\right)=\left(x_{n_{h}} \gamma_{n_{h}}, x_{n_{h}}^{\prime}\left[x_{n_{h}}, \gamma_{n_{h}}\right]\right) *\left(\Gamma \times \Gamma^{\prime}\right) \text {. }
$$

Puisque l'espace $N^{\prime} / \Gamma^{\prime}$ est compact, on peut trouver une sous-suite $\left(m_{h}\right)$ de $\left(n_{h}\right)$ et une suite $\left(\gamma_{m_{h}}^{\prime}\right)$ dans $\Gamma^{\prime}$ telles que la suite $\left(x_{m_{h}}^{\prime}\left[x_{m_{h}}, \gamma_{m_{h}}\right] \gamma_{m_{h}}^{\prime}\right)$ converge dans $N^{\prime}$. 
On a $\left(x_{m_{h}}, x_{m_{h}}^{\prime}\right) *\left(\Gamma \times \Gamma^{\prime}\right)=\left(x_{m_{h}} \gamma_{m_{h}}, x_{m_{h}}^{\prime}\left[x_{m_{h}}, \gamma_{m_{h}}\right] \gamma_{m_{h}}^{\prime}\right) *\left(\Gamma \times \Gamma^{\prime}\right)$. Finalement, la suite $\left(x_{m_{h}}, x_{m_{h}}^{\prime}\right) * \Gamma \times \Gamma^{\prime}$ converge dans $M /_{\Gamma \times \Gamma^{\prime}}$. Ceci prouve que $M / \Gamma_{\times \times \Gamma^{\prime}}$ est compact. Preuve de (4). La nil-transformation $S_{x}$ est définie par

$$
S_{x}\left(\left(y, y^{\prime}\right) *\left(\Gamma \times \Gamma^{\prime}\right)\right)=\left(a y[a, x],[a, y] y^{\prime}\right) *\left(\Gamma \times \Gamma^{\prime}\right) .
$$

Grâce au théorème de Green, on sait que $S_{x}$ est ergodique si et seulement si, pour tout caractère $\sigma$ de $M$, on a: si $\sigma(a[a, x], e)=1$ et si $\sigma$ vaut 1 sur $\Gamma \times \Gamma^{\prime}$, alors $\sigma$ vaut 1 sur $M$. On sait que l'ensemble des caractères $\sigma$ de $M$ qui valent 1 sur $\Gamma \times \Gamma^{\prime}$, ensemble qui est le dual du groupe compact abélien $M /_{M^{\prime} *\left(\Gamma \times \Gamma^{\prime}\right)}$, est dénombrable. Il nous suffit donc de montrer que, pour tout $\sigma \not \equiv 1$, tel que $\left.\sigma\right|_{\Gamma \times \Gamma^{\prime}} \equiv 1$,

$$
\{x \in N \mid \sigma(a[a, x] e)=1\} \quad \text { est négligeable. }
$$

Soit $\sigma$ un tel caractère de $M$. On pose, pour tout $y$ dans $N, \sigma_{1}(y)=\sigma(y, e)$ et, pour tout $y^{\prime}$ dans $N^{\prime}, \sigma_{2}\left(y^{\prime}\right)=\sigma\left(e, y^{\prime}\right)$. On a, pour tout $\left(y, y^{\prime}\right)$ dans $M$, $\sigma\left(y, y^{\prime}\right)=\sigma_{1}(y) \cdot \sigma_{2}\left(y^{\prime}\right)$. On remarque que $\sigma_{2}$ est un caractère de $N^{\prime}$ et que $\sigma_{2}$ vaut 1 sur $\Gamma^{\prime}$, cest à dire que $\sigma_{2}$ est un caractère du groupe compact abélien $N^{\prime} / \Gamma^{\prime}$. D'autre part, $\sigma_{1}$ vérifie, pour tous $z$ et $y$ dans $N$,

$$
\sigma_{1}(z) \cdot \sigma_{1}(y)=\sigma_{1}(z y) \cdot \sigma_{2}([z, y]) ; \text { de plus } \sigma_{1} \text { vaut } 1 \text { sur } \Gamma .
$$

Le groupe dérivé $M^{\prime}$ de $M$ est $\left\{\left(x^{\prime}, x^{\prime 2}\right) \mid x^{\prime} \in N^{\prime}\right\}$.

$$
\text { (En effet }\left(x, x^{\prime}\right) *\left(y, y^{\prime}\right) *\left(x, x^{\prime}\right)^{-1} *\left(y, y^{\prime}\right)^{-1}=\left([x, y],[x, y]^{2}\right) \text {.) }
$$

Le caractère $\sigma$ vaut bien sûr 1 sur $M^{\prime}$ et donc, pour tout $x^{\prime}$ dans $N^{\prime}$, on a $\sigma_{1}\left(x^{\prime}\right)=\left(\sigma_{2}\left(x^{\prime}\right)\right)^{-2}$. On en déduit que $\sigma((a[a, x], e))=1$ si et seulement si $\sigma_{1}(a)=$ $\sigma_{2}([x, a])^{2}$. L'application $x \mapsto \sigma_{2}([x, a])$ est un caractère de $N$, noté $\mathscr{X}$. Supposons que $\mathscr{X} \not \equiv 1$.

Le groupe $N$ étant connexe, le sous-groupe fermé $\operatorname{Ker} \mathscr{X}=\{x \in N \mid \mathscr{X}(x)=1\}$ est négligeable dans $N$. D'autre part $\mathscr{X}$ est surjectif et les ensembles du type $\{x \in N \mid \mathscr{X}(x)=\lambda\}$, où $\lambda$ est un complexe de module 1 , se déduisent de $\operatorname{Ker}(\mathscr{X})$ par translation. On en déduit que, pour tout $\lambda \in \mathbb{C}$,

$$
\{x \in N \mid \mathscr{X}(x)=\lambda\} \text { est négligeable. }
$$

On a vérifié ainsi que, si $\mathscr{X} \not \equiv 1$, alors $m(\{x \in N \mid \sigma(a[a, x], e)=1\})=0$ (ici, $m$ désigne la mesure de Haar de $N$ ).

Examinons le cas où $\mathscr{Z} \equiv 1$. Soit $\gamma \in \Gamma$. Pour tout $y$ dans $N$, posons $\mathscr{X}_{\gamma}(y)=$ $\sigma_{2}([y, \gamma])$. On définit ainsi un caractère $\mathscr{X}_{\gamma}$ de $N$. Du fait que $\mathscr{X} \equiv 1$, on déduit que $\mathscr{X}_{\gamma}(a)=1$; du fait que $\sigma_{2}$ vaut 1 sur $\Gamma^{\prime}$, on déduit que $\mathscr{X}_{\gamma}$ vaut 1 sur $\Gamma$. Grâce à l'hypothèse d'ergodicité de la translation à gauche par a sur $N / \Gamma$ on en déduit que $\mathscr{X}_{\gamma} \equiv 1$. Ainsi, on a, pour tout $x$ dans $N$ et tout $\gamma$ dans $\Gamma$,

$$
\sigma_{2}([x, a])=1 \text { et } \sigma_{2}([x, \gamma])=1 \text {. }
$$

Fixons $x$. Posons, pour tout $y$ dans $N, \mathscr{X}_{x}(y)=\sigma_{2}([y, x])$. On définit ainsi un caractère $\mathscr{X}_{x}$ de $N$ qui vaut 1 au point $a$ et qui vaut 1 sur $\Gamma$. On en déduit comme précédemment que $\mathscr{X}_{x} \equiv 1$. Finalement, on obtient $\sigma_{2} \equiv 1$. Ceci entraîne que $\sigma_{1}$ est un caractère de $N$ qui vaut 1 sur $\Gamma$, et qui vérifie, pour tout $x$ dans $N, \sigma_{1}(a[a, x])=\sigma_{1}(a)$. Si $\sigma_{1} \not \equiv 1$, on a donc: $\{x \in N \mid \sigma(a[a, x], e)=1\}$ est vide. La preuve de l'affirmation (4) est achevée. 


\section{REFERENCES}

[1] L. Auslander, L. Green \& F. Hahn. Flows on homogeneous spaces. Ann. Math. Studies 53 (1963).

[2] A. Bellow \& V. Losert. The weighted pointwise ergodic theorem and the individual ergodic theorem along subsequences. Trans. A.M.S. 288 (1985), 307-345.

[3] J. P. Conze \& E. Lesigne. Théorèmes ergodiques pour des mesures diagonales. Bull. Soc. Math. France 112 (1984), 143-175.

[4] H. Furstenberg. Stationary processes and prediction theory. Ann. Math. Studies 44 (1960).

[5] H. Furstenberg. Strict ergodicity and transformations of the torus. Amer. J. Math. 83 (1961), 573-601.

[6] H. Furstenberg. The structure of distal flows. Amer. J. Math. 85 (1963), 477-515.

[7] H. Furstenberg. Disjointness in ergodic theory .... Math. Systems Theory 1 (1967), 1-49.

[8] E. Lesigne. Sur la convergence ponctuelle de certaines moyennes ergodiques. Note au C.R.A.S. 298, série I, (17) (1984), 425-428.

[9] A.I. Malce'v. On a class of homogeneous spaces. A.M.S. Trans. 39 (1951).

[10] J. C. Oxtoby. Ergodic sets. Bull. A.M.S. 58 (1952), 116-136.

[11] W. Parry. Dynamical systems on nilmanifolds. Bull. London Math. Soc. 2 (1970), 38-40.

[12] W. Parry. Ergodic properties of affine transformations and flows on nilmanifolds. Amer. J. Math. 91 (1969), 757-771.

[13] M. S. Raghunathan. Discrete Subgroups of Lie Groups. (Springer Verlag, Berlin, 1972).

[14] N. Wiener \& A. Wintner. Harmonic analysis and ergodic theory. Amer. J. Math. 63 (1941), 415-426. 